\title{
Purse-String Versus Linear Conventional Skin Wound Closure of an Ileostomy: A Randomized Clinical Trial
}

\author{
Mina Alvandipour, Babak Gharedaghi ${ }^{1}$, Hamed Khodabakhsh ${ }^{2}$, Mohammad Yasin Karami ${ }^{3}$ \\ Department of Colorectal Surgery, Mazandaran University of Medical Sciences, Sari; ${ }^{1}$ Department of General Surgery, Mazandaran University \\ of Medical Sciences, Sari; ${ }^{2}$ Department of Emergency Medicine, Mazandaran University of Medical Sciences, Sari; ${ }^{3}$ Student Research \\ Committee, Faculty of Medicine, Shiraz University of Medical Sciences, Shiraz, Iran
}

Purpose: Infection is one of the most frequent complications that can occur after ileostomy closure. The incidence of wound infection depends on the skin closure technique, but there is no agreement on the perfect closure method for an ileostomy wound. The aim of this study was to evaluate the incidence of infection, the patient's approval, and the patient's pain between purse-string closure (PSC) and the usual linear closure (LC) of a stoma wound.

Methods: This randomized clinical trial enrolled 66 patients who underwent a stoma closure from February 2015 to May 2015 in Sari Emam Khomeini Hospital. Patients were divided into 2 groups according to the stoma closing method: the PSC group $(n=34)$ and the LC group $(n=32)$. The incidences of infection for the 2 groups were compared, and the patients' satisfaction and pain with the stoma were determined by using a questionnaire.

Results: Infection occurred in 1 of 34 PSC patients (2.9\%) and in 7 of $32 \mathrm{LC}$ patients (21.8\%), and this difference was statistically significant $(\mathrm{P}=0.021)$. Patients in the PSC group were more satisfied with the resulting wound scar and its cosmetic appearance at one month and three months after surgery $(\mathrm{P}=0.043)$.

Conclusion: After stoma closure, PSC was associated with a significantly lower incidence of wound infection and greater patient satisfaction compared to LC. However, the healing period for patients who underwent PSC was longer than it was for those who underwent LC.

Keywords: Surgical stoma; Ileostomy; Closure; Infection; Patient satisfaction

\section{INTRODUCTION}

A stoma is commonly used in the management of colorectal cancer surgery, inflammatory bowel disease (ulcerative colitis), and low anterior resections of rectal cancer. Many complications can occur after ileostomy closure (obstruction, infection, necrosis, leakage, and iatrogenic incisional hernia) [1]. Stoma closure site infection (SCSI) is also a frequent crisis after ileostomy closure,

Received: January 9,2016 Accepted: July 14, 2016

Correspondence to: Mohammad Yasin Karami, M.D.

Department of General Surgery, Shiraz University of Medical Sciences,

Shiraz, Iran

Tel: +98-9171800710, Fax: +98-7132330724

E-mail: yasinkarami@gmail.com

(C) 2016 The Korean Society of Coloproctology

This is an open-access article distributed under the terms of the Creative Commons Attribution NonCommercial License (http://creativecommons.org/licenses/by-nc/4.0) which permits unrestricted non-

commercial use, distribution, and reproduction in any medium, provided the original work is properly cited. with a reported incidence varying from $0 \%$ to $41 \%$. It is associated with increased costs for healthcare services, hospital stays, medications, and nursery care, as well as with increased morbidity and poor quality of life [2-8].

The circumferential purse-string (PS) approximation technique, introduced by Banerjee [3], after an ileostomy closure has been associated with less wound infection and scar formation, as well as with better cosmetic effect and patient satisfaction A study by Sutton et al. [8] advanced this technique and reported on a short series of successful infection-free ileostomy reversals. The PS technique combines the concept of leaving the wound open to provide drainage and minimize SCSI while still providing some degree of wound opposition to minimize healing time. No data as to whether the routine use of this form of closure is suitable in the Middle-East population, especially the Iranian population, are available.

This prospective, randomized, controlled trial compared traditional linear closure (LC) with purse-string closure (PSC), with 
SCSI as the primary comparative factor and patient satisfaction with the surgery as a secondary comparative factor. The aim of this study was to compare the incidence of surgical site infection, the satisfaction, and the pain experienced by patients who had undergone either a circumferential PS approximation or a conventional LC of an ileostomy in the Iranian population.

\section{METHODS}

This prospective, parallel, not blinded, randomized, clinical trial was carried out in the Department of Colorectal and General Surgery of Mazandaran University of Medical Sciences between February 2015 and May 2015. All patients with colorectal cancer who had undergone a protective ileostomy after surgery, as well as patients with a benign condition, such as a stab wound of the abdomen, who required an ileostomy, were included in this study; patients who were under 18 years of age, who died or did not continue the follow-up, and who needed secondary surgery at other centers were excluded from study. Surgery was performed by 2 types of surgeons: colorectal surgeons, and general surgeons. All patients scheduled for elective closure of an ileostomy at the Department of Colorectal and General Surgery of Mazandaran University of Medical Sciences were approached to be included in the study. Randomization was performed by using incidental number sheets. Informed consent was obtained before randomization. Ethical approval was granted by the Ethics Commttee, Mazandaran University of Medical Sciences (Registration Code: RCT2015040714483N3, www.IRCT.ir).

Patients were divided into 2 groups based on the closure technique: the PSC and LC groups. All data on the patients were retrieved from medical records and were recorded in a database. Analyzed variables included age, sex, body mass index (BMI), hemoglobin ( $\mathrm{Hb}$ ), blood pressure (BP), comorbidities of hyperlipidemia (HLP), diabetes mellitus (DM), or malignancy, hospital stay, time of surgery, and smoking status. Neither the patients nor the observer (surgeon) were told which of the methods had been used. The trial arm allocation would, however, have been clear on simple inspection of the wound by an observer who was aware of the differences between the techniques, so this cannot be considered a truly blinded trial. This is an inevitable and insoluble problem in this type of study. The patient populations in the 2 arms of the trial were well matched in most respects: sex, age, BMI, smoking status, original indication for surgery, and others. True 'blinding' in this trial was not possible.

We used the Patient and Observer Scar Assessment Scale (POSAS) developed by Draaijers et al. [9] The patient component of the POSAS contains seven questions: one each (six total) applying to scar-related pain, pruritus, color, thickness, relief, and pliability, and one applying to the patient's overall opinion of the scar [10]. Each of these is scored on a scale of 1-10, with 1 representing the best scar or sensation and 10 representing the worst. In this trial, only the patient component of the POSAS was applied because the secondary goal was to evaluate the patients' subjective view and satisfaction with the cosmetic outcome.

An elliptical incision was performed around the stoma to release the ileal loop. A skin-cut spindle-shape was used in both the LC and PSC groups, with a 1- to 2-mm margin from the ileostomy. In both groups, an end-to-end anastomosis (in 2 layers, polydioxanone [PDS], Prolene 3/0 sutures) was done, and the fascia was closed with figure-eight PDS 1/0 sutures; then, the skin was closed with interrupted nylon 3/0 sutures in the LC group, but with vicryl 0/2 sutures using a subcuticular technique in the PSC group. The wound opening was about 3 to $4 \mathrm{~mm}$ in diameter. Ninetyday morbidity and mortality were reviewed using medical records, outpatient clinic notes and the hospital's database.

The primary endpoint measure was SCSI as identified by a surgeon in the outpatient clinic within 30 days of surgery. A SCSI is defined as any infection of the superficial or deep tissues or the organ/space affected by the surgery when no prosthesis has been implanted) [11], and an infection is defined by the Center for Disease Control as the presence of at least one of the following conditions-purulent drainage, with or without laboratory confirmation, from the superficial incision and organisms isolated from an aseptically obtained culture of fluid from the superficial incision, and the presence of at least one of the following signs or symptoms of infection-pain or tenderness, localized swelling, redness, a superficial incision deliberately opened by surgeon unless the incision is culture negative, and a diagnosis of a superficial incisional SCSI by the surgeon or attending physician [11]. The secondary endpoint measures assessed were operating time, length of hospital stay, postoperative pain, and patient satisfaction with the wound or scar. Postoperative pain and patient satisfaction with the wound or scar were assessed by using a visual analog scale (VAS) $0-10$, with 0 indicating no pain and 10 the worst possible pain, at 3 days, 7 days, 1 month and 3 months after the surgery.

Follow-up was performed for all patients on postoperative days 1 and 3 in the hospital, at postoperative day 7 at the point of hospital discharge, and at postoperative days 30 and 90 after surgery. Wounds were inspected by an observer (surgeon) at all points of follow-up. Patient satisfaction and cosmetic outcome were assessed by a surgeon at the 30 -day visit and the 90 -day visit. In addition, at the 90-day visit, patients were asked about signs of SCSI and about pain and general satisfaction with the site of the scar.

Statistical analyses were performed using IBM SPSS Statistics ver. 21.0 (IBM Co., Armonk, NY, USA), setting statistical significance at $\mathrm{P}<0.05$. Chi-square or Mann-Whitney tests were used for the univariate analyses when appropriate. In addition, multivariate analyses of small vaiables were performed.

\section{RESULTS}

During the study period, February 2015 through May 2015, 72 patients who had undergone an elective ileostomy reversal were en- 
rolled. Three patients were excluded because of postoperative new stoma creation or insufficient follow-up (Fig. 1). Of the remaining 69 patients, 36 patients underwent a PSC and 33 underwent a LC. All patients received the treatment to which they had been randomly assigned. In the PSC group, 2 patients were excluded due to loss during follow-up, and in the LC group, one was excluded. The trial was stopped when the target numbers of subjects for the analysis that had completed the 90-day follow-up had been reached. Overall, $47 \%$ of the group was female and $53 \%$ was male.

Demographic variables, such as age and sex, and other variables, such as BMI, Hb, BP, comorbities of HLP, DM, and malignancy, hospital stay, duration of surgery and smoking status were recorded. All comorbidity and demographic data are presented in Table 1. No intraoperative or anastomosis complications were observed in either group. No significant differences between the 2 groups in sexual structure $(\mathrm{P}=0.80$, chi-square test), age $(\mathrm{P}=$ 0.893, Student $t$-test $), B M I(P=0.813$, Student $t$-test $)$, and $\mathrm{Hb}$ ( $\mathrm{P}$

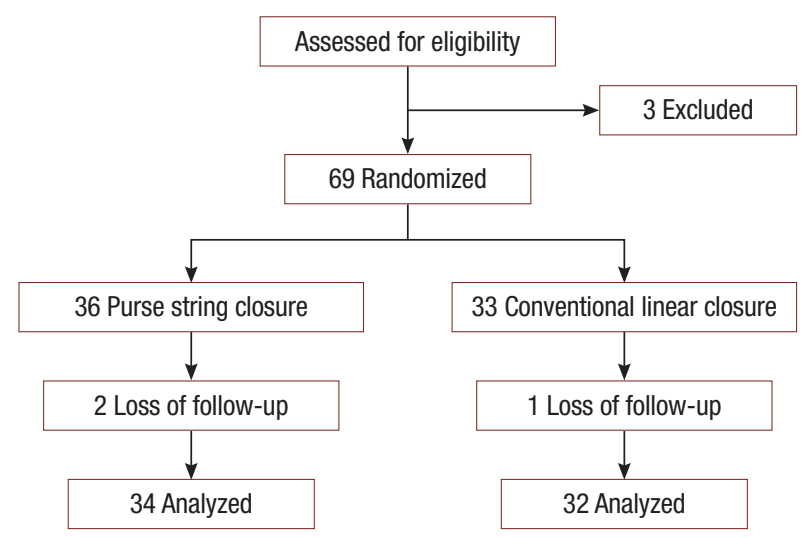

Fig. 1. Participation flow chart.

Table 1. Patients' demographic and operative-outcome charactristics

\begin{tabular}{lccc}
\hline Variable & $\begin{array}{c}\text { Purse string } \\
(\mathrm{n}=34)\end{array}$ & $\begin{array}{c}\text { Conventional linear } \\
(\mathrm{n}=32)\end{array}$ & P-value \\
\hline Age $(\mathrm{yr})$ & $52.85 \pm 13.95$ & $53.31 \pm 13.66$ & 0.893 \\
Sex ratio, male : female & 1.25 & 1 & 0.800 \\
Body mass index $\left(\mathrm{kg} / \mathrm{m}^{2}\right)$ & $24.3 \pm 2.34$ & $24.43 \pm 2.56$ & 0.813 \\
Hemoglobin (mg/dL) & $11.52 \pm 1.4$ & $11.83 \pm 1.41$ & 0.380 \\
Diabetes mellitus & $1 / 34(2.9)$ & $2 / 32(6.3)$ & 0.608 \\
Hypertension & $7 / 34(20.6)$ & $8 / 32(25)$ & 0.772 \\
Hyperlipidemia & $7 / 34(20.6)$ & $4 / 32(12.9)$ & 0.516 \\
Smoking & $3 / 34(9.1)$ & $5 / 32(15.6)$ & 0.475 \\
Malignancy & $24 / 34(70.6)$ & $17 / 32(53.1)$ & 0.200 \\
Hospital stay (day) & $6.55 \pm 1.18$ & $6.78 \pm 1.53$ & 0.512 \\
Duration of surgery (min) & $71.76 \pm 9$ & $69.84 \pm 10$ & 0.416 \\
Wound infection & $1 / 34(2.9)$ & $7 / 32(21.8)$ & 0.021 \\
\hline
\end{tabular}

Values are presented as mean \pm standard deviation or number (\%).
$=0.38$, Student $\mathrm{t}$-test).

Twenty-four persons (24 of 34, 70.6\%) in the PSC group had some kind of colorectal cancer versus 17 (17 of 32, 53.1\%) in the LC group, but this difference was not statistically significant $(\mathrm{P}=$ 0.20 , chi-square test). The mean hospital stays were $6.55 \pm 1.8$ and $6.78 \pm 1.53$ days in the PSC and the LC group, respectively, but this difference was not statistically significant $(\mathrm{P}=0.512)$. In the PSC group, the underlying disease for the ileostomy was malignant tumors in 24 patients (70.6\%) and benign diseases in 10 patients (29.4\%). In the CL group, it was malignant tumors in $17 \mathrm{pa}-$ tients (53.1\%) and nonmalignant diseases in 14 patients (46.9\%). However, the differences between the 2 groups were not statistically significant $(P=0.20)$. The duration of surgery was higher in the PSC group than it was in the LC group, but the difference was not statistically significant $(\mathrm{P}=0.416)$.

SCSI was evaluated in the 2 groups by using clinical signs of infection. No sign of SCSI was observed in either of the 2 groups on postoperative day 1; however, the infection rate in the CL group was statistically higher than it was in the PSC group on polstoperative days 3 and 7 ( $21.9 \%$ vs. $2.9 \% ; \mathrm{P}=0.022$; Fisher test). No sign of infection in either of the 2 groups was observed at either the 1or the 3-month follow-up (Figs. 2, 3).

Pain severity was assessed by using the VAS and was evaluated by using generalized linear models. The severity of pain in the
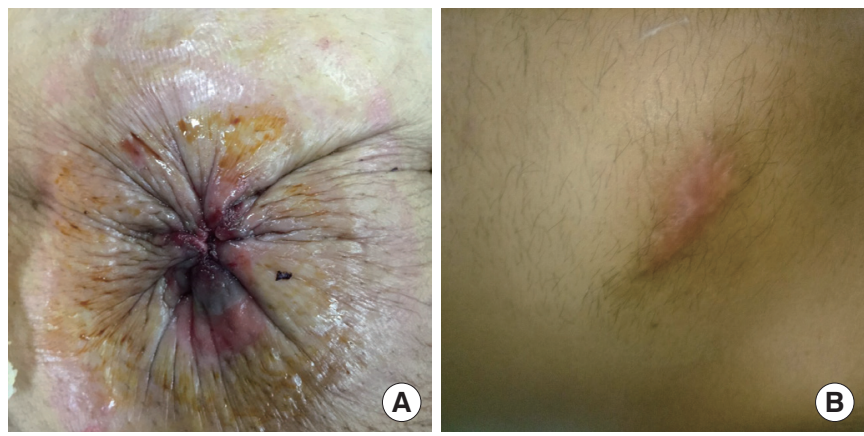

Fig. 2. Purse string suture (PSS) 1 day (A) and 3 months (B) after closure of the ileostomy.
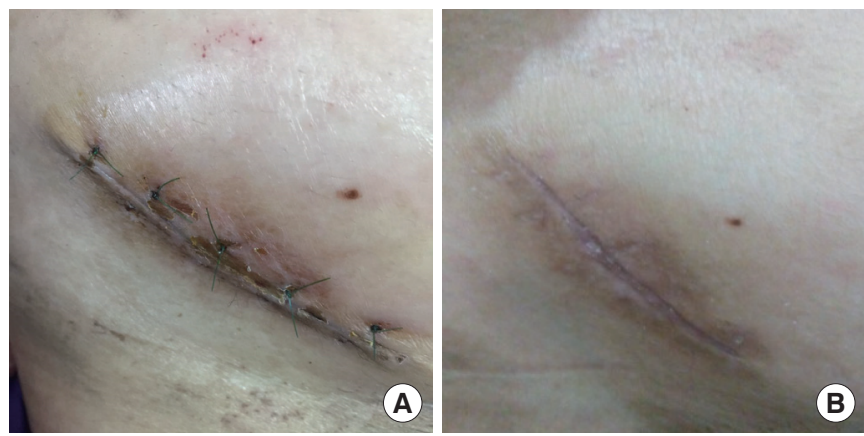

Fig. 3. Linear closure (LC) 10 days (A) and 3 months (B) after closure of the ileostomy. 
PSC group was $0.27 \%$ less than it was in the LC group (totally, mean \pm standard deviation [range], $2 \pm 1.2$ [1-7], Mann-Whitney test), a statistically significant difference. The mean ranks of pain were 30.78 in the PSC group and 36.39 in the LC group at postoperative day one, which was statistically significant $(P=0.002)$. Also, the mean rank of pain in the PSC group was significantly less than it was in the LC during the first 7 days after surgery, but after that, the values in both groups were similar. Satisfaction was assessed by using the POSAS and was evaluated by using generalized linear models (Mann-Whitney test). The mean ranks of satisfaction at postoperative days $1,3,7,30$, and 90 were significantly higher in the PSC group than in the LC group $(\mathrm{P}<0.001)$ (Fig. 4).

\section{DISCUSSION}

In this randomized, controlled trial, we found a significantly lower rate of stoma site infection associated with the PSC compared with the conventional LC ( $2.9 \%$ vs. $21.9 \%, \mathrm{P}=0.022)$. Our results are consistent with two recent, smaller, randomized, controlled trials $[12,13]$ comparing the PSC and the LC techniques after stoma reversal. Reid et al. [14] randomly assigned 61 ileostomy patients to either a PSC or an LC groups and found a significantly lower SCSI rate in the PSC group (6.7\% vs. $38.7 \%, \mathrm{P}=$ $0.005)$. The study sample size had been estimated at 60 patients, yet 66 were enrolled, and the trial was halted early because of an overwhelming number of SCSIs in the LC group. In addition, no significant differences were noted in baseline characteristics of the patients $(53.1 \%$ of the patients had cancer in the LC group vs. $70.6 \%$ in the PSC group, $\mathrm{P}=0.2$ ) that were not accounted for in a multivariable analysis. Camacho-Mauries et al. [12] randomly assigned 61 patients with ileostomies or colostomies to PSC or LC group. They found no SCSIs in the PSC group compared with a $36.6 \%$ SCSI rate in the LC group $(\mathrm{P}<0.0001)$ [13]. Sample size

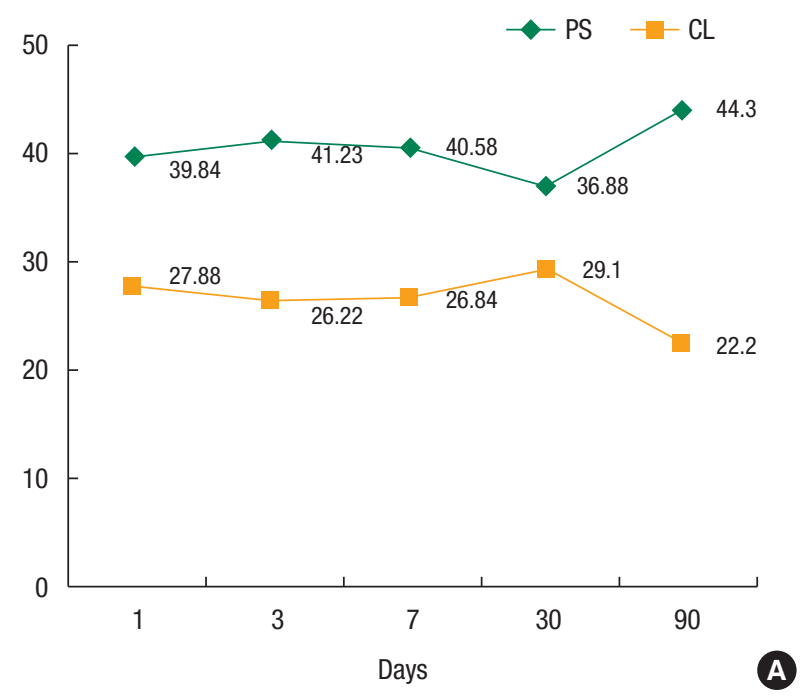

estimates or SCSI rates used in the calculations were not reported. Surgical site infection rates in the LC group in both the aforementioned studies were among the highest reported in the literature for this technique [6].

In our study, the SCSI rate of $21.9 \%$ in the LC group falls in the middle range of the rates in previous reports [8, 14-19] with an adequate sample size and a multivariable analysis to adjust for potential confounders. Our study confirms that the SCSI rate at the stoma site is significantly higher with LC than with PSC, and we believe these results to be generalizable to all patients who have ileostomy or colostomy reversals. Our data are also consistent with 2 recent systematic reviews that included randomized, controlled trials, as well as other case-control and retrospective reviews [20, 21]. Patients' satisfaction scores were rather high and were different between the 2 groups at 1 month and 3 months postoperatively. The mean rank of satisfaction in the LC group was higher on postoperative days 1,3 , and 7 , but at postoperative month 1 and month 3, the mean rank of satisfaction in the PSC group was higher than it was in the LC group had more scar formation than PS group. We mean more cosmetic change in ps group than LC group. Exchanging a stoma for a scar, of whatever type, is probably well-accepted by most patients, and this may have positively skewed the reported satisfaction scores in our study. A long-term assessment may have revealed differences in cosmetic outcomes, but this was beyond the scope of this study. Similar to our study, Reid et al. [14] found no differences between the groups, but Camacho-Mauries et al. [12] found higher patient satisfaction scores in the PSC group (very satisfied, 70\% in the PSC group vs. very satisfied, $20 \%$ in the LC group, $\mathrm{P}=0.0001$ ). In our study, all isolated stoma SCSIs were managed bedside by opening the incision to allow fluid drainage. No SCSI of the stoma site required a reoperation or additional antibiotic therapy. Similarly, in a study by Vermulst et al. [5], SCSIs at the stoma site were easily managed

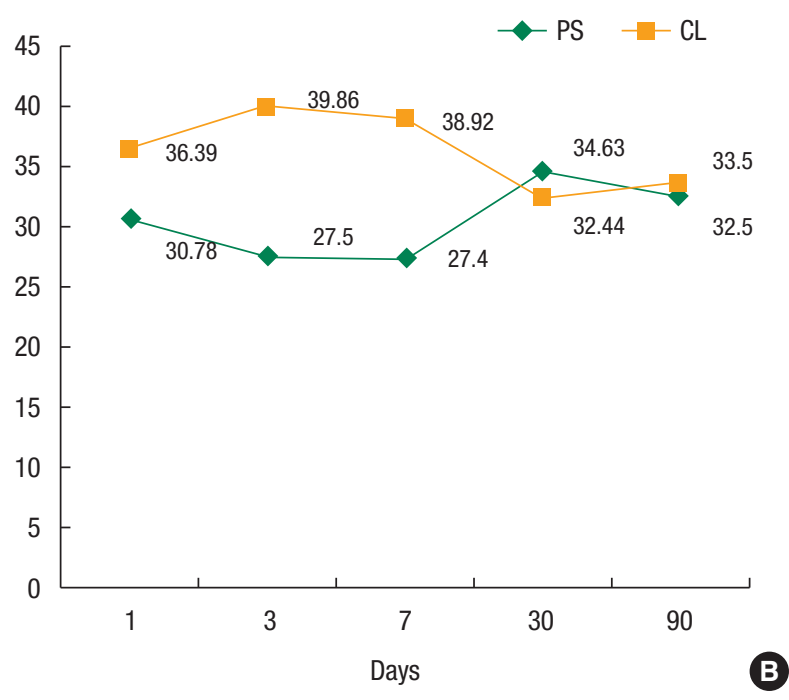

Fig. 4. Satisfaction (A) and pain (B) at days 1, 3, 7, 30, and 90. PS, purse-string; CL, conventional linear. 
without complications.

The biological process of wound healing consists of a series of complex interactions between cells, cytokines, and the extracellular matrix [22-24]. Sometimes, the healing time is relatively long due to seroma formation. The small orifice in the center of the wound allowed self drainage in the PSC group, so wound healing occurred more quickly due to less seroma formation. Murray et al. [25] retrospectively compared 25 patients with Conventional Linear (CL) to 37 patients with open-wound healing and found no increase in hospital stay, number of incisional hernias, or fistula formation between the 2 groups despite an SCSI rate of $36 \%$ in the CL group. However, in these studies, including ours, the length of follow-up may not have been sufficient to exclude an increased incidence of incisional hernias, which is a known late complication of an SCSI.

This study was limited by the variability in procedures performed by the 5 surgeons, the low sample size, the limited followup of 90 days, and the inability to perform blinding. True "blinding' in this trial was not possible due to the differences in the surgical methods of the 2 treatments and in the wound's appearance to the classifiers of infection. Potential procedural variability among the 5 surgeons involved in this study was possible, even though all surgeons were given precise instructions and a demonstration in the operating room before the study of the one technique each was to use. In addition, we did not record long-term complications such as incisional hernias or chronic nonhealing wounds and fistulae. These factors may affect the final cost of care linked to the treatment of SCSIs following stoma closure and should be studied in the future.

In conclusion, we found that the CL technique had a significantly lower incidence of SCSI than the CL closure technique. We could also show a difference in the incidence of patient satisfaction between groups. The purse string (PS) technique had a higher patient satisfaction than the CL technique, although wounds could take longer to heal when the PS approach is used. Our results suggest that the PS closure is the technique of choice to minimize SCSI after stoma reversal in the Iranian population.

\section{CONFLICT OF INTEREST}

No potential conflict of interest relevant to this article was reported.

\section{ACKNOWLEDGMENTS}

We would like to express our sincere gratitude to Mazandaran University of Medical Sciences. This study was extracted from the postgraduate thesis of Babak Gharedaghi, M.D., that was approved by Mazandaran University of Medical Sciences. The authors would also like to thank Dr. Mehrnoosh Maalhagh for editorial assistance.

\section{REFERENCES}

1. Tan WS, Tang CL, Shi L, Eu KW. Meta-analysis of defunctioning stomas in low anterior resection for rectal cancer. Br J Surg 2009; 96:462-72.

2. McGrath DR, Leong DC, Armstrong BK, Spigelman AD. Management of colorectal cancer patients in Australia: the National Colorectal Cancer Care Survey. ANZ J Surg 2004;74:55-64.

3. Banerjee A. Pursestring skin closure after stoma reversal. Dis Colon Rectum 1997;40:993-4.

4. Hackam DJ, Rotstein OD. Stoma closure and wound infection: an evaluation of risk factors. Can J Surg 1995;38:144-8.

5. Vermulst N, Vermeulen J, Hazebroek EJ, Coene PP, van der Harst E. Primary closure of the skin after stoma closure. Management of wound infections is easy without (long-term) complications. Dig Surg 2006;23:255-8.

6. Lahat G, Tulchinsky H, Goldman G, Klauzner JM, Rabau M. Wound infection after ileostomy closure: a prospective randomized study comparing primary vs. delayed primary closure techniques. Tech Coloproctol 2005;9:206-8.

7. Chow A, Tilney HS, Paraskeva P, Jeyarajah S, Zacharakis E, Purkayastha $S$. The morbidity surrounding reversal of defunctioning ileostomies: a systematic review of 48 studies including 6,107 cases. Int J Colorectal Dis 2009;24:711-23.

8. Sutton CD, Williams N, Marshall LJ, Lloyd G, Thomas WM. A technique for wound closure that minimizes sepsis after stoma closure. ANZ J Surg 2002;72:766-7.

9. Draaijers LJ, Tempelman FR, Botman YA, Tuinebreijer WE, Middelkoop E, Kreis RW, et al. The patient and observer scar assessment scale: a reliable and feasible tool for scar evaluation. Plast Reconstr Surg 2004;113:1960-5.

10. Vercelli S, Ferriero G, Sartorio F, Stissi V, Franchignoni F. How to assess postsurgical scars: a review of outcome measures. Disabil Rehabil 2009;31:2055-63.

11. Williams LA, Sagar PM, Finan PJ, Burke D. The outcome of loop ileostomy closure: a prospective study. Colorectal Dis 2008;10:4604.

12. Camacho-Mauries D, Rodriguez-Diaz JL, Salgado-Nesme N, Gonzalez QH, Vergara-Fernandez O. Randomized clinical trial of intestinal ostomy takedown comparing pursestring wound closure vs conventional closure to eliminate the risk of wound infection. Dis Colon Rectum 2013;56:205-11.

13. Alexander JW, Solomkin JS, Edwards MJ. Updated recommendations for control of surgical site infections. Ann Surg 2011;253: 1082-93.

14. Reid K, Pockney P, Pollitt T, Draganic B, Smith SR. Randomized clinical trial of short-term outcomes following purse-string versus conventional closure of ileostomy wounds. British Journal of Surgery 2010 Oct 1;97(10):1511-7.

15. Harold DM, Johnson EK, Rizzo JA, Steele SR. Primary closure of stoma site wounds after ostomy takedown. Am J Surg 2010;199: 621-4. 
16. Lee JR, Kim YW, Sung JJ, Song OP, Kim HC, Lim CW, et al. Conventional linear versus purse-string skin closure after loop ileostomy reversal: comparison of wound infection rates and operative outcomes. J Korean Soc Coloproctol 2011;27:58-63.

17. Marquez TT, Christoforidis D, Abraham A, Madoff RD, Rothenberger DA. Wound infection following stoma takedown: primary skin closure versus subcuticular purse-string suture. World J Surg 2010;34:2877-82.

18. Amin SN, Memon MA, Armitage NC, Scholefield JH. Defunctioning loop ileostomy and stapled side-to-side closure has low morbidity. Ann R Coll Surg Engl 2001;83:246-9.

19. Lewis P, Bartolo DC. Closure of loop ileostomy after restorative proctocolectomy. Ann R Coll Surg Engl 1990;72:263-5.

20. Li LT, Hicks SC, Davila JA, Kao LS, Berger RL, Arita NA, et al. Circular closure is associated with the lowest rate of surgical site infection following stoma reversal: a systematic review and multiple treatment meta-analysis. Colorectal Dis 2014;16:406-16.

21. McCartan DP, Burke JP, Walsh SR, Coffey JC. Purse-string ap- proximation is superior to primary skin closure following stoma reversal: a systematic review and meta-analysis. Tech Coloproctol 2013;17:345-51.

22. Zekavat O, Amanat A, Karami M, Paydar S, Gramizadeh B, Zareian-Jahromi M. Wound Healing Studies Using Punica granatum Peel: An Animal Experimental Study. Adv Skin Wound Care 2016;29:217-25.

23. Karami MY, Zekavat OR, Amant A. Excisional wound healing activity of carboxymethyle cellulose in diabetic rat. J Jahrom Univ Med Sci 2012;9:48-57.

24. Jahanabadi S, Karami MY, Paydar S, Farzinnia H, Ghobadifar MA, Mansournia N, et al. Albusite: a novel synthetic gel for promotion of skin wound healing in rats. Online J Biol Sci 2015;15: 104-10.

25. Murray BW, Cipher DJ, Pham T, Anthony T. The impact of surgical site infection on the development of incisional hernia and small bowel obstruction in colorectal surgery. Am J Surg 2011; 202:558-60. 